\title{
ACL Reconstruction in High School and College-Aged Athletes: Does Autograft Choice Influence ACL Revision Rates?
}

\author{
MOON Knee Group SpindlerKurt P.1HustonLaura J.2ZajichekAlexander3ReinkeEmily \\ K.4AmendolaAnnunziato4Andrish Jack T.1BrophyRobert H.5DunnWarren R.6FlaniganDavid \\ C.7JonesMorgan H.1KaedingChristopher C.7MarxRobert G.8MatavaMatthew \\ J.5McCartyEric C.9ParkerRichard D.1VidalArmando F.9WolcottMichelle L.9WolfBrian \\ R.10WrightRick W.5 \\ ${ }^{1}$ Department of Orthopaedic Surgery, Cleveland Clinic Foundation, Cleveland, $\mathrm{OH}$ \\ ${ }^{2}$ Vanderbilt Orthopaedic Institute, Vanderbilt University Medical Center, Nashville, TN \\ ${ }^{3}$ Department of Quantitative Health Sciences, Cleveland Clinic Foundation, Cleveland, $\mathrm{OH}$ \\ ${ }^{4}$ Department of Orthopaedic Surgery, Duke University, Durham, NC \\ ${ }^{5}$ Department of Orthopaedic Surgery, Washington University School of Medicine at Barnes- \\ Jewish Hospital, St. Louis, MO \\ ${ }^{6}$ Department of Orthopaedics, Reedsburg Hospital, Reedsburg, WI \\ ${ }^{7}$ Department of Orthopaedic Surgery, The Ohio State University School of Medicine, Columbus, \\ $\mathrm{OH}$ \\ ${ }^{8}$ Department of Orthopaedics, Hospital for Special Surgery, New York, NY \\ ${ }^{9}$ Department of Orthopaedic Surgery, University of Colorado School of Medicine, Denver, CO \\ ${ }^{10}$ Department of Orthopaedic Surgery, University of lowa School of Medicine, lowa City, IA
}

\section{Abstract}

Background: Physicians' and patients' decision-making process between bone-patellar tendonbone (BTB) versus hamstring autografts for anterior cruciate ligament (ACL) reconstruction (ACLR) may be influenced by a variety of factors in the young, active athlete.

Hypothesis/Purpose: The purpose was to determine the incidence of both ACL graft revision rates and contralateral ACL tears resulting in subsequent ACLR in a cohort of high school and college-aged athletes who initially underwent either a primary BTB or hamstring autograft ACLR.

Study Design: Prospective longitudinal cohort study

Methods: Study inclusion criteria were patients aged 14-22 who were injured in sport, had a contralateral normal knee, and were due to have a unilateral primary ACLR with either a BTB or hamstring autograft. All patients were prospectively followed for six years to determine whether any subsequent ACLR surgery had occurred to either knee since their initial ACLR. Multivariable

Corresponding Author: Kurt P. Spindler, MD, Department of Orthopaedics, Cleveland Clinic Foundation, 5555 Transportation Blvd., Garfield Heights, OH 44125, spindlk@ ccf.org. 
regression modeling controlled for age, sex, ethnicity/race, body mass index, sport and competition level, baseline activity level, knee laxity, and graft type. The 6-year outcomes were the incidence of subsequent ACLR to either knee.

Results: Eight hundred thirty-nine (839) patients were eligible, of which 770 (92\%) had 6-year follow-up for the primary outcome measure of incidence of subsequent ACLR surgery. The median age was 17, with $48 \%$ females, and the distribution of BTB to hamstring was 492 (64\%) and $278(36 \%)$ respectively.

The incidence of subsequent ACLR at 6 years was 9.2\% in the ipsilateral knee, $11.2 \%$ in the contralateral normal knee, and $19.7 \%$ for either knee. High-grade preoperative knee laxity (odds ratio [OR]: 2.4; 95\% CI: 1.4,3.9; $\mathrm{p}=0.001$ ), autograft type (OR: $2.1 ; 95 \%$ CI: $1.3,3.5 ; \mathrm{p}=0.004$ ), and age (OR: $0.8 ; 95 \% \mathrm{CI}: 0.7,0.96 ; \mathrm{p}=0.009$ ) were the 3 most influential predictors of an ACLR graft revision on the ipsilateral knee. The odds of an ACLR graft revision were 2.1 times higher for patients receiving a hamstring autograft than patients receiving a BTB autograft (95\% CI: 1.3, 3.5; $\mathrm{p}=0.004$ ). No significant differences were found between autograft choice when looking at incidence of subsequent ACL reconstructions in the contralateral knee.

Conclusions: There is a high incidence of both ACLR graft revisions and contralateral normal ACL tears resulting in subsequent ACLRs in this young athletic cohort. The incidence of ACL graft revisions at 6 years following index surgery is 2.1 times higher with hamstring autograft compared to a BTB autograft.

\section{Keywords}

anterior cruciate ligament; ACL reconstruction; ACL revision; failure; outcomes; autograft

\section{INTRODUCTION}

An ongoing debate continues between the autograft choice of bone-tendon-bone (BTB) versus hamstrings in terms of which reduces the risk of recurrent ligament disruption. Systematic reviews, meta-analyses, and a 2011 Cochrane database review on randomized controlled trials (RCTs) between these autograft choices for anterior cruciate ligament (ACL) reconstruction (ACLR) have reported no major clinically relevant differences in terms of graft failure rates. ${ }^{3}, 6,7,9,19,20,24,27,28,32,40,41$ However, these prior studies have failed to control for either sport-related variables (i.e., sport played, competition level) or other important risk factors (i.e., age, sex, pre-operative knee laxity levels), which some clinicians utilize when selecting between a BTB versus hamstring autograft.

Currently, the most frequently asked questions regarding autograft choice focus around our highly active/competitive athletes, with the majority in high school and college age range. Unfortunately, most previously published studies on graft failure rates have been performed over a wide age range or have included both highly competitive athletes and non-athletes. $8,12,16,17,26,28,30,31$ Additionally, no study to date has analyzed the influence of the degree of knee laxity (lachman, anterior drawer, and/or pivot shift) on outcomes for specific autograft types. A previously published report from Kaeding et al. looking at the predictors of subsequent ACL tears after ACLR did not restrict the age range of athletes injured in sports to 14-22 years old and did not model/control for competition level or knee laxity. ${ }^{16}$ 
Thus, to provide the highest evidence today on whether there is a difference between BTB or hamstring autograft in the incidence of recurrent ACL injury, potential risk factors should either be stratified in an RCT comparing graft types or controlled through multivariable analysis. The goal of this study was to determine the incidence of both ACL graft revision rates and contralateral ACL tears resulting in subsequent ACLR in a cohort of high school and college-aged athletes who initially underwent either a BTB or hamstring autograft primary ACLR. Our hypothesis was there would be no differences in failure between autograft types at 6 years following a patient's index ACL surgery.

\section{MATERIALS and METHODS}

\section{Setting and Study Population}

After obtaining approval from each site's respective institutional review boards, our multicenter consortium began enrolling patients in 2002. This consortium consisted of 7 sites with 17 surgeons over the multi-year enrollment period. One university functioned as the data coordinating center for the study and was responsible for entering baseline data and collecting follow-up data on all patients. The details of enrollment, patient-reported outcome capture, surgical documentation at ACLR surgery and method of follow-up have been described previously. ${ }^{4}$

This analysis included only young athletes between the ages of 14-22 years who were injured in while participating in a sport and were scheduled to have either a BTB or hamstring autograft primary ACLR. Study exclusions were patients not injured in sport (i.e., motor vehicle accidents, workman's compensation cases, etc.), having any previous surgery on either the ipsilateral or contralateral knee, intended allografts or hybrid grafts (autograft + allograft) for the ACLR, concomitant MCL/LCL/PCL surgical procedure, or a revision ACLR. In order to preserve degrees of freedom for our statistical model, we also eliminated cases that were sparsely populated with past variables of interest. As such, we excluded the few individuals who self-identified as married or were current smokers ( $\mathrm{n}=38$ cases). Figure 1 details the patient population inclusion/exclusion criteria and follow-up.

\section{Data Source and Measurement}

After informed consent was obtained, each participant completed a questionnaire encompassing baseline demographics, injury descriptors, sports participation level, comorbidities, surgical knee history, and patient-reported outcome measures that included the IKDC, ${ }^{14,} 15$ the 5 subscales of the KOOS (symptoms, pain, activities of daily living, sports/recreation, knee-related quality of life $)^{29}$ and the Marx activity rating scale. ${ }^{23}$ Each surgeon completed a detailed form that documented the results of the examination under anesthesia, the surgical technique, the arthroscopic findings and treatment of any concomitant meniscus and cartilage injuries. Of note, no patients underwent a physeal sparing technique, as all were deemed skeletally mature at the time of their surgery via preoperative radiographs. After surgery, the patients were given a uniform set of standardized, evidence-based rehabilitation guidelines. ${ }^{37-39}$ Completed baseline data forms were mailed from each participating site to the data coordinating center. Data from both the patient and surgeon questionnaires were scanned with Teleform software (OpenText, 
Waterloo, Ontario, Canada) using optical character recognition, and the scanned data were verified and exported to a master database. A series of logical error and quality control checks were subsequently performed prior to data analysis.

\section{Follow-up}

Six-year follow-up was completed by mail with re-administration of the same questionnaire which they completed at baseline. In addition, patients were contacted either by phone or by email to determine whether any ACL graft revision and contralateral ACL tears resulting in a subsequent ACLR were performed since their index ACL surgery. Every effort was made to obtain the operative note on these surgical procedures so that they could be accurately and consistently characterized.

\section{Quantitative Variables and Statistical Methods}

The original model from which these are based upon is described in Dunn et al..$^{5}$ As the intention of this analysis was to provide assistance to a physician in the office discussing autograft choice with a patient prior to ACLR surgery, we only included known variables at the time of the physician-patient encounter or during their preoperative exam. This excluded meniscus and articular cartilage injuries and treatment, which can only be confirmed at surgery where treatment decisions are rendered. We also were able to remove marital and smoking status from the model by excluding the few individuals that were not single and not current smokers. The 'heard a pop' variable was excluded, as we have grown less confident in the robustness of the patient report since the original analysis. Preoperative knee laxity was added to the models as was done in Magnussen et al. ${ }^{22}$ The variables analyzed were age, sex, ethnicity/race, body mass index (BMI), sport played at the time of injury (basketball, football, soccer, other), competition level (organized high school/club/college sport vs. recreational), baseline Marx activity level, and high-grade preoperative knee laxity at the time of ACLR (yes/no). High-grade knee laxity was determined by having either a Lachman or anterior drawer examination greater than a $10 \mathrm{~mm}$ difference from the contralateral side or a $3+$ pivot-shift during their examination under anesthesia. This definition has shown to be predictive of increased odds of having a subsequent revision ACL surgery. ${ }^{22}$ We hypothesized that preoperatively most surgeons recognize a "significantly lax knee" during their preoperative examination, especially given that some use this variable in their decision-making for which autograft to use prior to surgery in order to obtain appropriate informed consent.

Our two outcomes of interest were subsequent ACLR graft revision (ipsilateral knee) and subsequent contralateral normal ACL tear resulting in ACLR.

Univariate Summary-Continuous variables were calculated and summarized with medians (with 25\%, 75\% interquartile ranges [IQR]), and categorical variables with counts (with percentages [\%]). All baseline patient demographic characteristics, index ACLR information, and incidence of subsequent ipsilateral and contralateral ACLRs at 6 years were summarized. 
Modeling-For the outcomes of interest, multivariate models were built. Any missing baseline information was imputed using Multivariate Imputation by Chained Equations (MICE) via the R package. ${ }^{35}$

a. Predictors of Graft Type: It was first of interest to assess which variables influence the choice of graft to be used. One important adjustor of interest was the surgeon who performed the index ACLR surgery. Due to a large number of unique surgeons ( $\mathrm{n}=17)$, and that some surgeons performed relatively few cases enrolled in the study, a generalized linear mixed-model was used where the surgeon variable was treated as a random effect, producing the random-intercept logistic regression model. ${ }^{2}$

This model is similar to the standard logistic regression model, except that the variability across surgeons with respect to graft type was captured by an estimation of the variance of the random effects distribution (which is assumed to be normally distributed). The fixedeffects variables in the model were: age, sex, race, BMI, sport of injury, competition level, high-grade preoperative knee laxity, and baseline Marx activity level.

Bootstrap confidence intervals were obtained for all model parameters to assess statistical significance. The relative importance of each characteristic on predicting graft type was ranked by the amount of increase in Akaike's Information Criterion (AIC) upon removal from the full model, including the standard deviation of the random effects. AIC quantifies the amount of information explained by the covariates, while penalizing variables for the number of degrees of freedom they use. ${ }^{1}$ Models with a smaller AIC are preferred, therefore, a large increase in AIC after removal of a variable would reflect its importance.

b. Predictors of Subsequent ACL Reconstruction: The goal of this analysis was to determine the effect of graft type on the risk of a subsequent ACLR on either the ipsilateral or contralateral knee. The following variables were considered: age, sex, race, BMI, sport of injury, competition level, high-grade knee laxity, graft type, and baseline Marx activity level.

First, a random forest model was built to assess the functional form and interactivity of variables with respect to the risk of each outcome. The relationships between the outcomes and age and BMI were explored to determine if evidence on non-linear relationships existed. In addition, the interactivity of the graft type with the sport of injury and BMI were also explored as it is suspected that the graft type chosen for patients may depend on the sport of injury because of the perceived effect on the future outcomes. This preliminary analysis then allowed identification of potential relationships to be accounted for in an interpretable regression model without missing important effects.

Once potential interactions and non-linear relationships were found, a linear model was formulated. First, a standard logistic regression model was run with all covariates. Pearson residuals were then assessed to examine the potential for subsequent correlation within surgeons on the risk of a subsequent ACLR graft revision (ipsilateral knee) or the risk of the contralateral normal ACL tear resulting in an ACLR (contralateral knee). ${ }^{13}$ Pairs of distinct residuals were randomly sampled for each surgeon, and the correlation was computed. This was repeated 10,000 times to assess many pairs of observations. The comparison was made 
by both weighted and unweighted correlation estimates due to the imbalance in surgeon cases. An appropriate model was then used to account for additional variability across surgeons, if necessary.

The AIC of the full models were compared to the AIC of the model removing non-linear terms, interactions (if applicable), as well as race and competition level to reduce the model complexity to prevent over fitting. The relative importance of the variables in the final models were then ranked according to individual removal from the (potentially) reduced model. The predictive accuracy of the final models were assessed by the concordance probability (C-index) via the ".632 estimator". ${ }^{11}$ Odds ratios, $95 \%$ confidence intervals, and p-values were reported for the final models. Nomograms were also produced to get a more intuitive understanding of effect size.

\section{RESULTS}

\section{Study Population}

Of the eligible 839 patients meeting the inclusion/exclusion criteria, 6-year follow-up related to subsequent surgical outcomes was obtained on 770 patients (92\%; Figure 1). Table 1 displays univariate summaries of baseline demographic information of the patient cohort. The study population was $52 \%$ male, with a median age of 17 years and median BMI of 23.3. The high activity level of this cohort is reflected in the ceiling effect observed with a median baseline Marx score of 16 (maximum score $=16$ ). Although the vast majority of patients were involved in organized high school, club or college sports (84\%), the sport at the time of injury was evenly distributed across four categories: basketball (29\%), football (23\%), soccer (20\%), and other (28\%). Thirty-three percent (33\%) of patients in this young cohort were classified as having "high-grade" preoperative knee laxity. The distribution of BTB to hamstring autografts was $492(64 \%)$ and $278(36 \%)$, respectively.

\section{Predictors of Graft Type: Which Variables Affect Chosen Autograft Type?}

Figure 2 displays the relative variable importance of covariates in explaining the variability among the choice of autograft used for the index ACLR (BTB vs. hamstring). After adjusting for baseline characteristics, the surgeon performing the surgery had the largest influence on what graft was used.

Although surgeon choice had by far the most influence in determining which graft type was chosen for the index ACLR, the variables of competition level, age, existence of high-grade preoperative knee laxity, BMI, and race/ethnicity were also found to play a factor, albeit from a distant secondary value. Table 2 lists the confidence intervals for the odds of choosing a BTB versus hamstring autograft. We found that athletes with a competition level in high school/club/college were more likely to receive a BTB autograft than those competing recreationally. In addition, younger age, presence of high-grade preoperative knee laxity, lower BMI, and black athletes were more likely to receive a BTB autograft. 


\section{Is Surgeon a Surrogate for Graft Choice?}

Given that surgeon choice had the most influence in determining which graft type was chosen for the index ACLR, we wanted to ensure that surgeon was not a surrogate for graft choice. In order to accomplish this, a prediction model was constructed for each surgeon on the exact same hypothetical patients, except the only difference being the surgeon itself. Figure 3 displays the distributions of the predictions, stratified by surgeon. Regardless of patient characteristics, for example, there were 4 study surgeons (\#9, 7, 15, and 16 in the graph below) who will almost always choose a BTB autograft. Conversely, there were 4 study surgeons (\#2, 10, 5, and 4 in the graph below) who will almost always choose a hamstring autograft. The rest of the surgeons $(n=9)$ vary their graft choice, depending upon their individual decision algorithm. As such, it was determined that surgeon cannot always be assumed to be a surrogate for graft choice.

\section{Incidence of Subsequent Ipsilateral and Contralateral ACL Reconstructions at 6 Years}

Table 3 depicts the number of subsequent ipsilateral, contralateral, and combined ACLRs documented at 6 years in our young, active cohort. Approximately $9.2 \%$ of patients had a subsequent ipsilateral revision ACLR (7.1\% BTB, 13.0\% hamstrings), $11.2 \%$ had a subsequent contralateral ACL tear resulting in a primary ACLR $(12.6 \% \mathrm{BTB}, 8.6 \%$ hamstrings), and $19.7 \%$ had one or the other within 6 years of their index ACLR surgery.

\section{Prediction Models for Subsequent ACL Reconstruction (ipsilateral and contralateral knees)}

Figure 4 displays the relative variable importance of each predictor in the final model according to the increase in AIC upon removal. High-grade knee laxity, autograft type, and age were the three most influential and significant predictors of a subsequent ACL graft revision on the ipsilateral knee, respectively, whereas the sport of the index injury was the only significant predictor of a subsequent native ACL tear resulting in a primary ACLR on the contralateral knee.

Table 4 displays the odds ratios (with associated $95 \%$ confidence intervals) and p-values for each variable in the final models. The following statistically significant findings can be concluded. First, increasing age by 1 year results in a 16\% decrease in the odds of a subsequent ACL graft revision on the ipsilateral knee (similar effect for the contralateral knee, though not significant, with a p-value $=0.07$ ). Second, the odds of a patient not injured in basketball, football, or soccer to have a subsequent native ACLR on the contralateral knee is $~ 68 \%$ (CI: $35 \%$ to $84 \%$ ) less than those injured playing basketball. Third, the odds of a patient with high-grade knee laxity at baseline having a subsequent ACL graft revision on the ipsilateral knee is between 1.4 and 3.9 times that of patients without high-grade knee laxity (point estimate is 2.35). Fourth, sex, BMI, and baseline Marx activity did not show a significant association with the risk of recurrent a subsequent ACLR in either knee. Lastly, the odds of a subsequent ACL graft revision on the ipsilateral knee for patients receiving a hamstring autograft were 2.1 times higher than the odds of a patient receiving a BTB autograft (CI: 1.27, 3.54). Conversely, the odds of incurring a subsequent ACL tear on the contralateral knee for patients receiving a hamstring autograft were $68 \%$ lower than patients receiving a BTB autograft, but this difference was not significant ( $\mathrm{p}=0.144$; Table 4). 
To further illustrate the effect of each variable, Figures 5 and 6 display nomograms of each model. Figure 5 (for the ipsilateral knee) illustrates that, for otherwise low-risk patients (defined as a 5\% risk of ACL re-tear, independent of graft type), a hamstring graft can increase the risk of ACL graft revision from 5\% to 10\% (essentially doubling the risk). For example, based on the Figure 5 nomogram, an active 18-year old female soccer player with a BMI of 26 and classified as not having high-grade knee laxity will have a baseline nomogram score of 144 points (age $=18$ years [51 points]; $B M I=26$ [21 points]; sex=female [0 points]; sport at injury $=$ soccer $[42$ points]; high-grade knee laxity $=$ no [0 points]; Marx activity level=16=yes [30 points]). If this athlete underwent an ACLR with a BTB autograft (0 points), her nomogram score would remain at 144 points, which would correspond to an approximate 6-year risk of an ipsilateral ACL graft tear of 0.05 (5\%; Figure 5). However, if this same female soccer player underwent an ACLR with a hamstring autograft (55 points on the nomogram), her total nomogram score would increase to 199 points (144+55), which would correspond to an approximate 6-year ipsilateral graft retear risk of 0.10 (10\%; Figure $5)$.

Conversely, for otherwise high-risk patients (defined as an approximate 30\% risk of ACL retear, independent of graft type), a hamstring graft can increase the risk of ACL graft revision from $30 \%$ to approximately 50\% (an approximate 50\% increase). For example, based on the Figure 5 nomogram, an active 14-year old male football player with a BMI of 26 and classified as having high-grade knee laxity will have a baseline nomogram score of 290 points (age $=14$ years $[100$ points]; $B M I=26$ [21 points]; sex=male [29 points]; sport at injury = football [ 48 points]; high-grade knee laxity=yes [62 points]; Marx activity level=16=yes [30 points]). If this athlete underwent an ACLR with a BTB autograft (0 points), his nomogram score would remain at 290 points, which would correspond to an approximate 6year risk of an ipsilateral ACL graft tear of approximately 0.29 (29\%; Figure 5). However, if this same male football player underwent an ACLR with a hamstring autograft (55 points on the nomogram), his total nomogram score would increase to 345 points $(290+55)$, which would correspond to an approximate 6-year ipsilateral graft retear risk of 0.46 (46\%; Figure $5)$.

\section{DISCUSSION}

We found a high rate of subsequent ACLRs on both the ipsilateral and contralateral knees in our 14-22 year-old athletes who had either a hamstring or BTB autograft index ACLR. Our predictive modeling suggests a 2.1 times higher recurrent ipsilateral ACL graft revision rate with hamstring autografts compared to BTB autografts.

Surgeons are the most influential factor in choosing whether a patient was likely to have a BTB or a hamstring autograft. Secondary factors included a patient's competition level and their age.

This is consistent with the findings of Wright et al., who determined that surgeon choice was the leading factor in what type of graft (autograft or allograft) a patient was given for a revision ACLR. ${ }^{10}$ 
At 6 years follow-up, our overall incidence of subsequent ACLR in either knee was 19.7\% (152/770) in this young athletic cohort. Interestingly, when stratified by ipsilateral and contralateral sides, divergent results were noted. For the ipsilateral index knee, there was a 9.2\% failure rate at 6 years, with BTB grafts undergoing a lower incidence of subsequent ACLRs compared to hamstrings (7.1\% vs. 13\%). Conversely, patients with an initial BTB autograft underwent higher subsequent ACL tears resulting in a primary ACLR in their contralateral knee compared with patients with a hamstring autograft $(12.6 \%$ vs. $8.6 \%)$, for an overall $11.2 \%$ contralateral ACLR rate. After controlling for the aforementioned risk factors (age, sex, BMI, sport, competition level, presence of high-grade laxity), we found that the odds of an ACLR graft revision on the ipsilateral knee for patients receiving a hamstring autograft were 2.1 times higher than the odds of a patient receiving a BTB autograft (95\% CI: 1.3, 3.5; $\mathrm{p}=0.004)$. There was no significant difference found between autograft choices when looking at incidence of subsequent ACLR in the contralateral knee.

Previous studies have reported higher failure rates in hamstring autografts compared to BTB autografts. ${ }^{8,12,26,28,30}$ In a Level 1 systematic review, Reinhardt et al. pooled six studies across all age groups to evaluate graft failure. ${ }^{28}$ The overall failure rate for the hamstring group was $15.8 \%$ (26/165), while the subsequent failure rate for the patellar tendon group was $7.2 \%$ (11/153). Gifstad et al. pooled 45,998 primary ACL reconstructions from the Scandinavian ACL registries and showed that over a 5-year follow-up period, patients who received a hamstring autograft had a statistically higher risk of revision compared with patients who had received a BTB autograft $(4.2 \%$ vs. $2.8 \%$; $\mathrm{p}<0.001) .{ }^{8}$ Similarly, Persson et al. reviewed over 12,000 patients undergoing primary ACL reconstruction from the 20042012 Norwegian Cruciate Ligament Registry and found that patients with a hamstring autograft had more than twice the risk of revision compared with patients with a BTB autograft (5.1\% vs. $2.1 \%) .{ }^{26}$ Ho et al. reported on 561 ACLRs with an average patient age of 15.4 years (range: 5 to 19 years) and found that soft tissue grafts were twice as likely to fail compared to BTB grafts (13\% vs. $6 \%$; $\mathrm{p}<0.001) .{ }^{12}$ These findings were consistent with our current study, in that young patients receiving hamstring autografts were more likely to experience a subsequent ACLR in their ipsilateral knee (13.0\%), as compared to those receiving a BTB autograft (7.1\%). Like Ho's study, the cohort that we assessed is novel because it only includes the highest risk patients-young, active individuals injured in sports. This patient population has the highest rate of recurrent ligament disruption after ACLR, and re-injury is a devastating complication for the competitive athlete. Therefore, both patients and their surgeons have a strong interest in being able to make an informed decision about the optimal autograft choice to provide the best outcome.

Previous studies have reported that younger age is a predictor for higher subsequent ACL graft failures. ${ }^{16,17,26,31,34,36}$ In a systematic review and meta-analysis, Wiggins et al. pooled 19 studies to look at secondary injury rates amongst an ACL reconstructed population. ${ }^{36}$ Overall, the ACL reinjury rate was $15 \%$ (ipsilateral - 7\%; contralateral - 8\%), while the combined ACL reinjury rate for patients younger than 25 years of age jumped to $21 \%$. They concluded that age (patients who were younger than 25 years old) and returning to a high level of activity were important risk factors associated with secondary ACL injury. In Persson et al.'s study, they found that younger age was the most important risk factor for undergoing an ACL revision. ${ }^{26}$ In a study of 2683 individuals (mean age of 27 years) with 
unilateral primary ACLRs from the MOON cohort, Kaeding et al. reported higher activity level and younger age to be associated with higher recurrent ligament injury rates for either ACL reconstructed or contralateral knees. ${ }^{16}$ However, they did not limit their cohort to highschool or college-aged athletes. Webster et al. evaluated a cohort of 750 patients (mean age of 28.5 years) and noted lower age to be associated with increased risk of recurrent ligament disruption on both the ACL reconstructed and contralateral side. ${ }^{34}$ This group was unable to model failure rates comparing graft types because their cohort consisted of $97 \%$ hamstring autografts.

Our analysis was aimed at utilizing data that would be available to a clinician trying to make a preoperative decision about which graft to select to provide the individual patient with the best possible outcome in terms of incidence of ACL tears to either knee. Therefore, the models that we constructed included data about demographics and history (age, sex, race, BMI, sports participation and level of competition, activity level) and clinical exam (preoperative knee laxity). While we could have constructed more complex models that potentially could explain more variation and were better predictors of outcome, we would have lost the ability to provide a useful tool for patients and their surgeons to use during the preoperative decision-making process.

This study is not without limitations. Preoperative knee laxity was not quantified via instrumentation, but by the study surgeon using the IKDC classification system. Previous studies have demonstrated some variability when classifying pre-operative knee laxity utilizing this system. ${ }^{18,25,33}$ The current study had variability across our surgeons in the proportions of patients classified with high-grade preoperative knee laxity. It is unknown whether these differences represent variation among surgeons or differences in their individual patient populations. ${ }^{21}$ However, these assessments represent the current method of laxity measurement most frequently used when physicians make treatment decisions. Thus, the data is generalizable. Lastly, there are other possible risk factors for subsequent ACLR that this study did not collect (i.e. return to sport comparison between groups), so early return to sport or other underlying factors may have contributed to systematic differences between the group who received a BTB versus hamstring autograft.

\section{CONCLUSIONS}

This study evaluated a unique cohort of patients -- high school and college-aged athletes engaged in sports -- to assess the factors associated with differences in subsequent ACLR rates between BTB versus hamstring autografts. There is a relatively high incidence of subsequent ACLRs in both the ipsilateral and contralateral knees in this young athletic cohort at six years. Multivariable analysis demonstrated that the incidence of subsequent ACL graft revisions at 6 years following index surgery is 2.1 times higher with hamstring autograft compared to BTB autograft.

\section{REFERENCES}

1. Akaike H Information theory as an extension of the maximum likelihood principle-In: Second International Symposium on Information Theory (Eds) Petrov BN, Csaki F. BNPBF Csaki. Budapest: Academiai Kiado 1973. 
2. Bates D, Mächler M, Bolker B, Walker S. Fitting Linear Mixed-Effects Models Using lme4. 2015 2015;67(1):48.

3. Biau DJ, Tournoux C, Katsahian S, Schranz PJ, Nizard RS. Bone-patellar tendon-bone autografts versus hamstring autografts for reconstruction of anterior cruciate ligament: meta-analysis. BMJ. 2006;332(7548):995-1001. [PubMed: 16603564]

4. Cox CL, Huston LJ, Dunn WR, et al. Are articular cartilage lesions and meniscus tears predictive of IKDC, KOOS, and Marx activity level outcomes after anterior cruciate ligament reconstruction? A 6-year multicenter cohort study. Am J Sports Med. 2014;42(5):1058-1067. [PubMed: 24647881]

5. Dunn WR, Spindler KP. Predictors of activity level 2 years after anterior cruciate ligament reconstruction (ACLR): a Multicenter Orthopaedic Outcomes Network (MOON) ACLR cohort study. Am J Sports Med. 2010;38(10):2040-2050. [PubMed: 20709944]

6. Forster MC, Forster IW. Patellar tendon or four-strand hamstring? A systematic review of autografts for anterior cruciate ligament reconstruction. Knee. 2005;12(3):225-230. [PubMed: 15911297]

7. Gabler CM, Jacobs CA, Howard JS, Mattacola CG, Johnson DL. Comparison of Graft Failure Rate Between Autografts Placed via an Anatomic Anterior Cruciate Ligament Reconstruction Technique: A Systematic Review, Meta-analysis, and Meta-regression. Am J Sports Med. 2015.

8. Gifstad T, Foss OA, Engebretsen L, et al. Lower risk of revision with patellar tendon autografts compared with hamstring autografts: a registry study based on 45,998 primary ACL reconstructions in Scandinavia. Am J Sports Med. 2014;42(10):2319-2328. [PubMed: 25201444]

9. Goldblatt JP, Fitzsimmons SE, Balk E, Richmond JC. Reconstruction of the anterior cruciate ligament: meta-analysis of patellar tendon versus hamstring tendon autograft. Arthroscopy. 2005;21(7):791-803. [PubMed: 16012491]

10. Group M. Factors Influencing Graft Choice in Revision Anterior Cruciate Ligament Reconstruction in the MARS Group. J Knee Surg. 2016;29(6):458-463. [PubMed: 26588108]

11. Hastie TJ, Tibshirani RJ, Friedman JH. The elements of statistical learning : data mining, inference, and prediction : with 200 full-color illustrations. New York: Springer; 2001.

12. Ho B, Edmonds EW, Chambers HG, Bastrom TP, Pennock AT. Risk Factors for Early ACL Reconstruction Failure in Pediatric and Adolescent Patients: A Review of 561 Cases. J Pediatr Orthop. 2018;38(7):388-392. [PubMed: 27379789]

13. Højsgaard S, Halekoh U, Yan J. The R Package geepack for Generalized Estimating Equations. 2005 2005;15(2):11.

14. Irrgang JJ, Anderson AF, Boland AL, et al. Development and validation of the international knee documentation committee subjective knee form. Am J Sports Med. 2001;29(5):600-613. [PubMed: 11573919]

15. Irrgang JJ, Anderson AF, Boland AL, et al. Responsiveness of the International Knee Documentation Committee Subjective Knee Form. Am J Sports Med. 2006;34(10):1567-1573. [PubMed: 16870824]

16. Kaeding CC, Pedroza AD, Reinke EK, Huston LJ, Spindler KP. Risk Factors and Predictors of Subsequent ACL Injury in Either Knee After ACL Reconstruction: Prospective Analysis of 2488 Primary ACL Reconstructions From the MOON Cohort. Am J Sports Med. 2015;43(7):15831590. [PubMed: 25899429]

17. Kamien PM, Hydrick JM, Replogle WH, Go LT, Barrett GR. Age, graft size, and Tegner activity level as predictors of failure in anterior cruciate ligament reconstruction with hamstring autograft. Am J Sports Med. 2013;41(8):1808-1812. [PubMed: 23813800]

18. Lange T, Freiberg A, Droge P, Lutzner J, Schmitt J, Kopkow C. The reliability of physical examination tests for the diagnosis of anterior cruciate ligament rupture--A systematic review. Man Ther. 2015;20(3):402-411. [PubMed: 25466498]

19. Li S, Chen Y, Lin Z, Cui W, Zhao J, Su W. A systematic review of randomized controlled clinical trials comparing hamstring autografts versus bone-patellar tendon-bone autografts for the reconstruction of the anterior cruciate ligament. Arch Orthop Trauma Surg. 2012;132(9):12871297. [PubMed: 22661336]

20. Li S, Su W, Zhao J, et al. A meta-analysis of hamstring autografts versus bone-patellar tendonbone autografts for reconstruction of the anterior cruciate ligament. Knee. 2011;18(5):287-293. [PubMed: 20850327] 
21. Magnussen RA, Reinke EK, Huston LJ, Group M, Hewett TE, Spindler KP. Effect of High-Grade Preoperative Knee Laxity on Anterior Cruciate Ligament Reconstruction Outcomes. The American Journal of Sports Medicine. 2016;44(12):3077-3082. [PubMed: 27480978]

22. Magnussen RA, Reinke EK, Huston LJ, Group M, Hewett TE, Spindler KP. Factors Associated With High-Grade Lachman, Pivot Shift, and Anterior Drawer at the Time of Anterior Cruciate Ligament Reconstruction. Arthroscopy. 2016;32(6):1080-1085. [PubMed: 26821957]

23. Marx RG, Stump TJ, Jones EC, Wickiewicz TL, Warren RF. Development and evaluation of an activity rating scale for disorders of the knee. Am J Sports Med. 2001;29(2):213-218. [PubMed: 11292048]

24. Mohtadi NGH, Chan DS, Dainty KN, Whelan DB. Patellar tendon versus hamstring tendon autograft for anterior cruciate ligament rupture in adults. Cochrane Database of Systematic Reviews. 2011(9).

25. Peeler J, Leiter J, MacDonald P. Accuracy and reliability of anterior cruciate ligament clinical examination in a multidisciplinary sports medicine setting. Clin J Sport Med. 2010;20(2):80-85. [PubMed: 20215888]

26. Persson A, Fjeldsgaard K, Gjertsen JE, et al. Increased risk of revision with hamstring tendon grafts compared with patellar tendon grafts after anterior cruciate ligament reconstruction: a study of 12,643 patients from the Norwegian Cruciate Ligament Registry, 2004-2012. Am J Sports Med. 2014;42(2):285-291. [PubMed: 24322979]

27. Poolman RW, Abouali JA, Conter HJ, Bhandari M. Overlapping systematic reviews of anterior cruciate ligament reconstruction comparing hamstring autograft with bone-patellar tendon-bone autograft: why are they different? J Bone Joint Surg Am. 2007;89(7):1542-1552. [PubMed: 17606794]

28. Reinhardt KR, Hetsroni I, Marx RG. Graft selection for anterior cruciate ligament reconstruction: a level I systematic review comparing failure rates and functional outcomes. Orthop Clin North Am. 2010;41(2):249-262. [PubMed: 20399364]

29. Roos EM, Roos HP, Lohmander LS, Ekdahl C, Beynnon BD. Knee Injury and Osteoarthritis Outcome Score (KOOS)--development of a self-administered outcome measure. J Orthop Sports Phys Ther. 1998;28(2):88-96. [PubMed: 9699158]

30. Samuelsen BT, Webster KE, Johnson NR, Hewett TE, Krych AJ. Hamstring Autograft versus Patellar Tendon Autograft for ACL Reconstruction: Is There a Difference in Graft Failure Rate? A Meta-analysis of 47,613 Patients. Clin Orthop Relat Res. 2017;475(10):2459-2468. [PubMed: 28205075]

31. Sanders TL, Pareek A, Hewett TE, et al. Long-term rate of graft failure after ACL reconstruction: a geographic population cohort analysis. Knee Surg Sports Traumatol Arthrosc. 2017;25(1):222228. [PubMed: 27522592]

32. Spindler KP, Kuhn JE, Freedman KB, Matthews CE, Dittus RS, Harrell FE Jr. Anterior cruciate ligament reconstruction autograft choice: bone-tendon-bone versus hamstring: does it really matter? A systematic review. Am J Sports Med. 2004;32(8):1986-1995. [PubMed: 15572332]

33. Tanaka M, Vyas D, Moloney G, Bedi A, Pearle AD, Musahl V. What does it take to have a highgrade pivot shift? Knee Surg Sports Traumatol Arthrosc. 2012;20(4):737-742. [PubMed: 22222616]

34. Webster KE, Feller JA, Leigh WB, Richmond AK. Younger patients are at increased risk for graft rupture and contralateral injury after anterior cruciate ligament reconstruction. Am J Sports Med. 2014;42(3):641-647. [PubMed: 24451111]

35. White IR, Royston P, Wood AM. Multiple imputation using chained equations: Issues and guidance for practice. Stat Med. 2011;30(4):377-399. [PubMed: 21225900]

36. Wiggins AJ, Grandhi RK, Schneider DK, Stanfield D, Webster KE, Myer GD. Risk of Secondary Injury in Younger Athletes After Anterior Cruciate Ligament Reconstruction: A Systematic Review and Meta-analysis. Am J Sports Med. 2016;44(7):1861-1876. [PubMed: 26772611]

37. Wright RW, Haas AK, Anderson J, et al. Anterior Cruciate Ligament Reconstruction Rehabilitation: MOON Guidelines. Sports Health. 2015;7(3):239-243. [PubMed: 26131301]

38. Wright RW, Preston E, Fleming BC, et al. A systematic review of anterior cruciate ligament reconstruction rehabilitation: part I: continuous passive motion, early weight bearing, 
postoperative bracing, and home-based rehabilitation. J Knee Surg. 2008;21(3):217-224. [PubMed: 18686484]

39. Wright RW, Preston E, Fleming BC, et al. A systematic review of anterior cruciate ligament reconstruction rehabilitation: part II: open versus closed kinetic chain exercises, neuromuscular electrical stimulation, accelerated rehabilitation, and miscellaneous topics. J Knee Surg. 2008;21(3):225-234. [PubMed: 18686485]

40. Xie X, Liu X, Chen Z, Yu Y, Peng S, Li Q. A meta-analysis of bone-patellar tendon-bone autograft versus four-strand hamstring tendon autograft for anterior cruciate ligament reconstruction. Knee. 2015;22(2):100-110. [PubMed: 25547048]

41. Xie X, Xiao Z, Li Q, et al. Increased incidence of osteoarthritis of knee joint after ACL reconstruction with bone-patellar tendon-bone autografts than hamstring autografts: a metaanalysis of 1,443 patients at a minimum of 5 years. Eur J Orthop Surg Traumatol. 2015;25(1):149159. [PubMed: 24748500] 
What is known about the subject:

Young athletes face a high rate of recurrent ACL injury after ACLR.

What this study adds to existing knowledge:

While many studies have looked at subsequent ACL failure rates in young athletes, this study incorporates only primary autograft ACLRs (BTB versus hamstring) and controls for a host of known risk factors (i.e., age, sex, body mass index (BMI), sport played at the time of injury, competition level, and high-grade preoperative knee laxity) when evaluating subsequent ACLRs in either knee in a group of high school and college athletes. When controlling for these factors, ACLR graft revisions were approximately 2fold higher with hamstring autografts as compared to BTB autografts. 


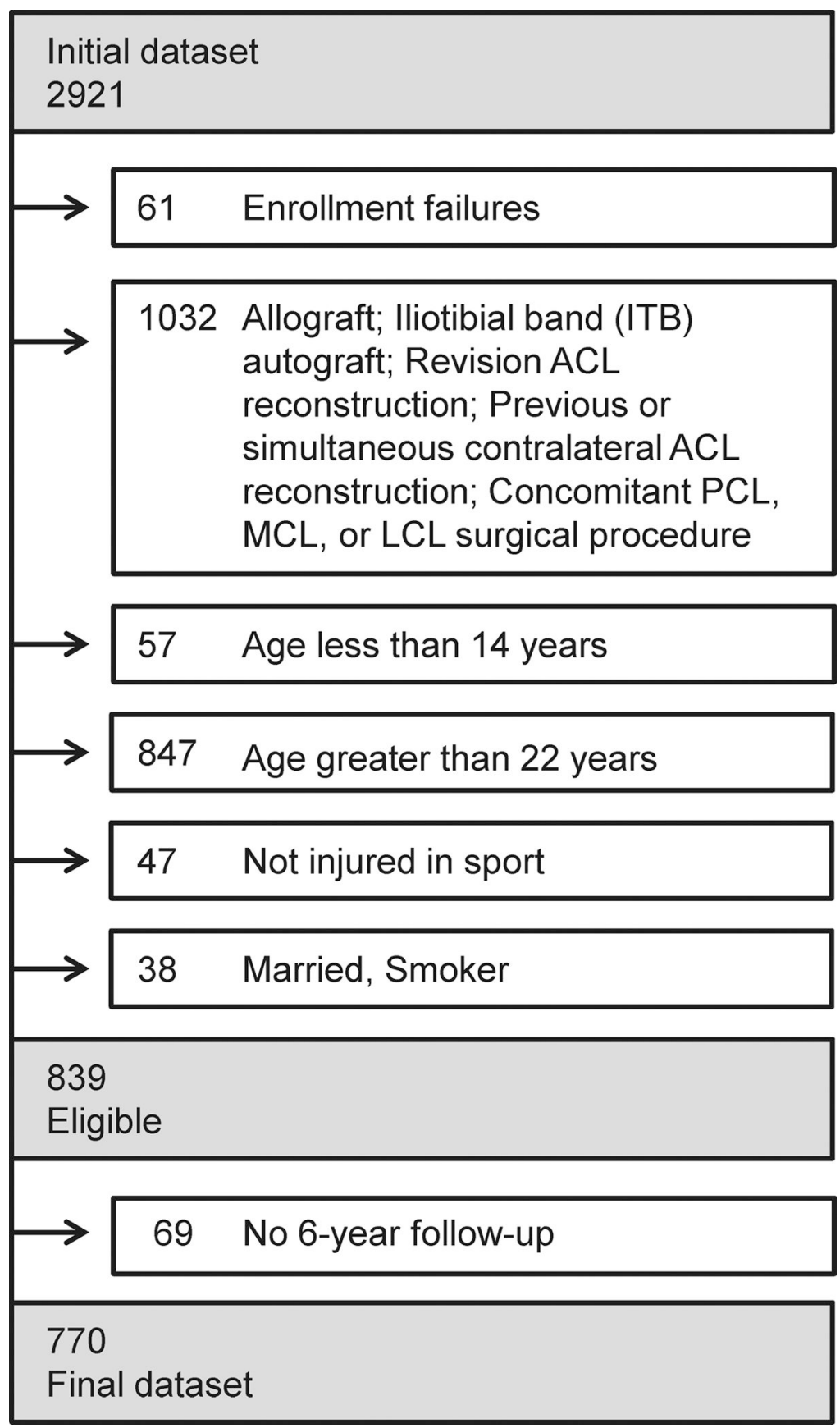

Figure 1.

Enrollment Flow Diagram 


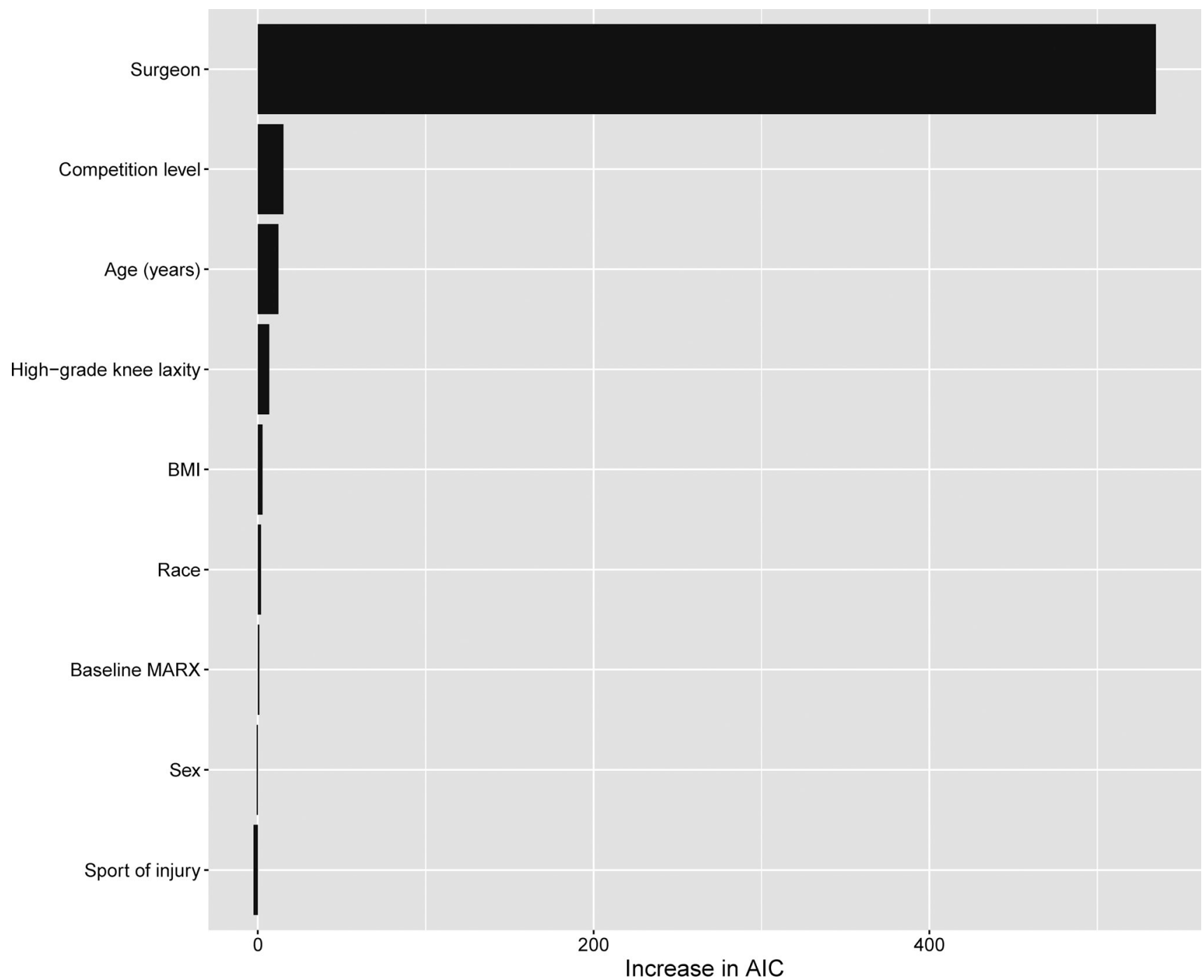

Figure 2.

Relative variable importance in explaining variability in autograft type used for the index ACL reconstruction. 


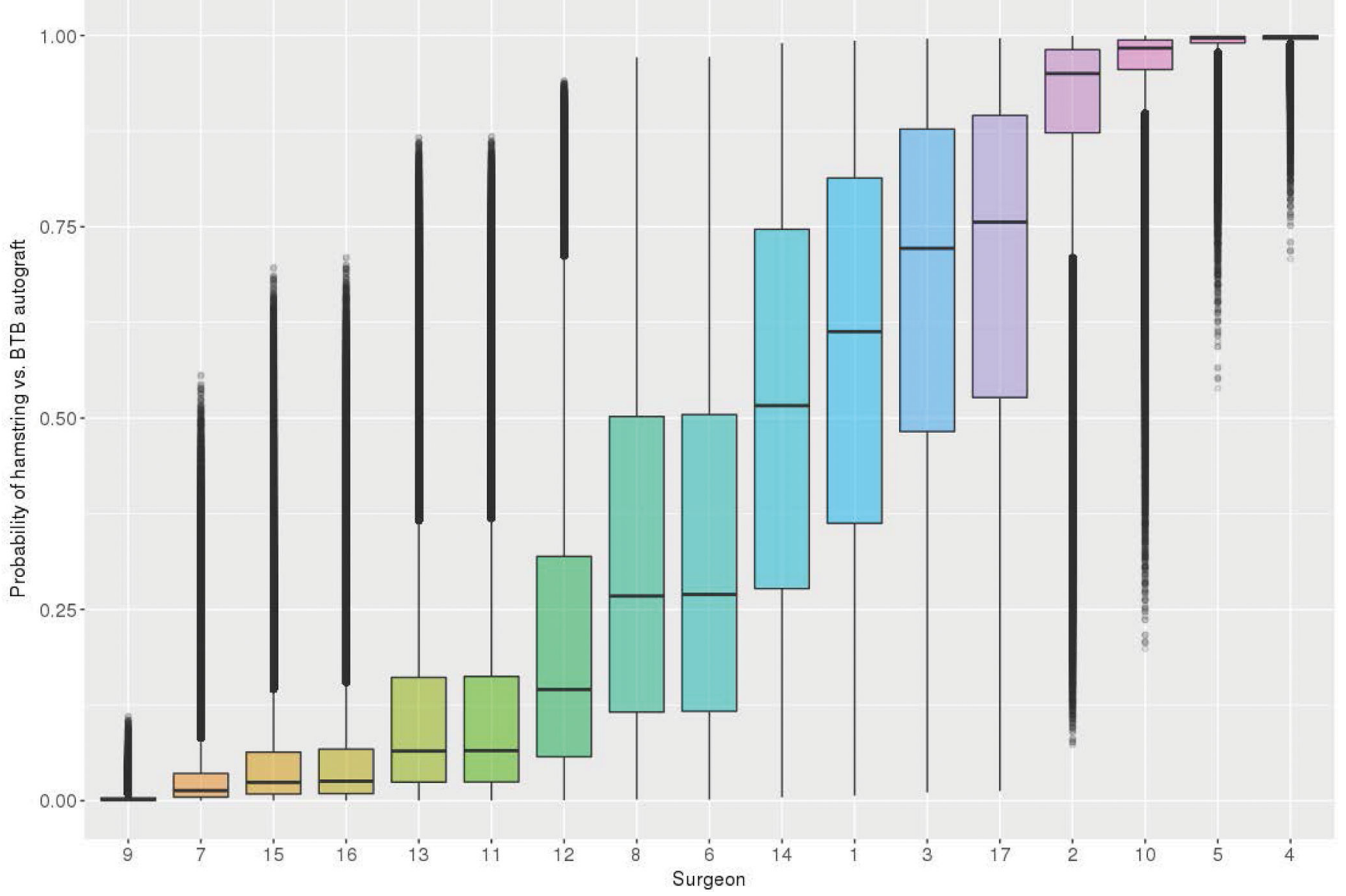

Figure 3.

Distributions of the probability of choosing a hamstring autograft for all combinations of observed baseline characteristics, stratified by study surgeon. A probability near 0 depicts a surgeon who will rarely, if ever, choose a hamstring autograft. Conversely, a probability near 1.00 depicts a surgeon who will always choose a hamstring autograft, no matter what the circumstances. Key: BTB = bone-patellar tendon-bone. 

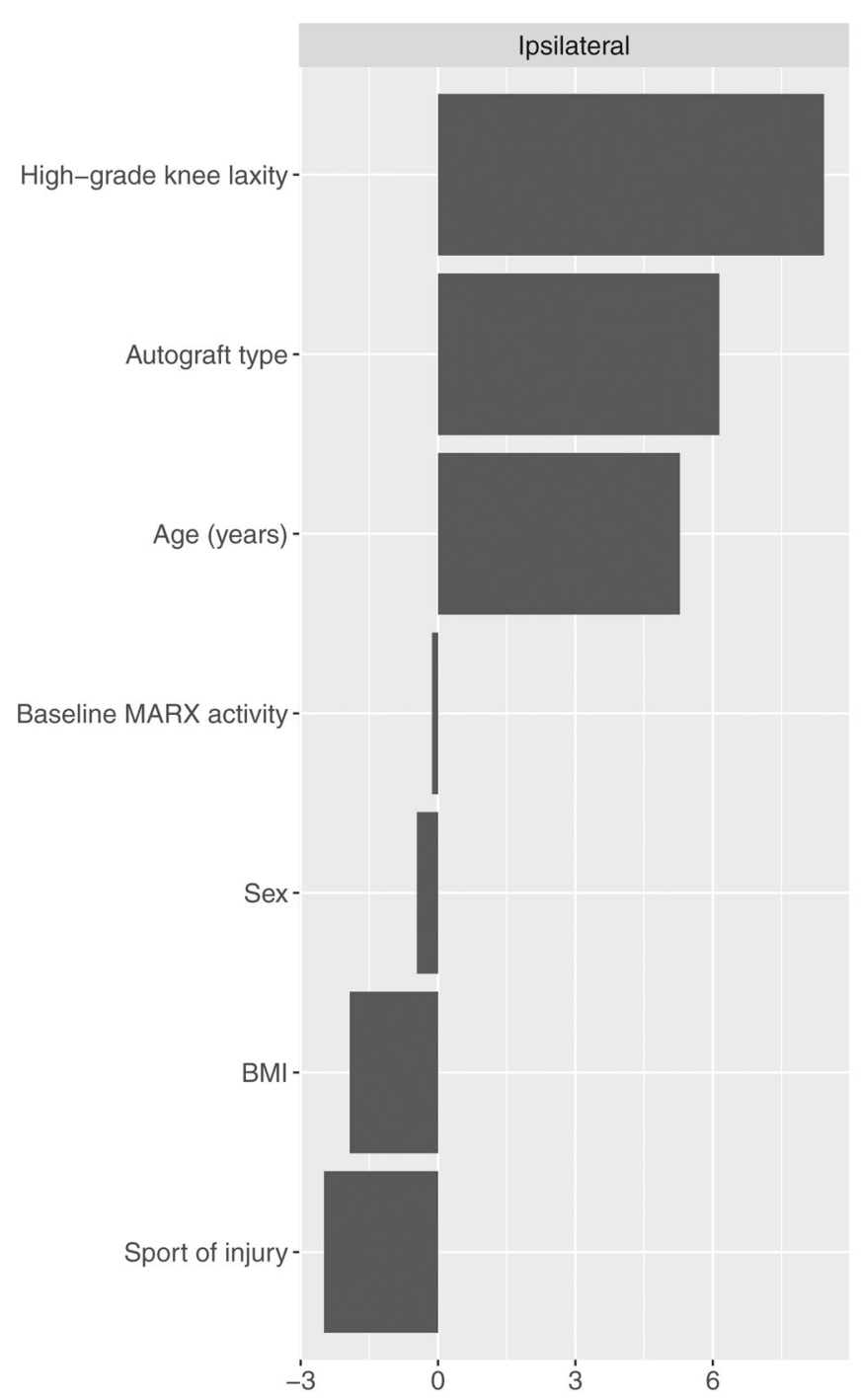

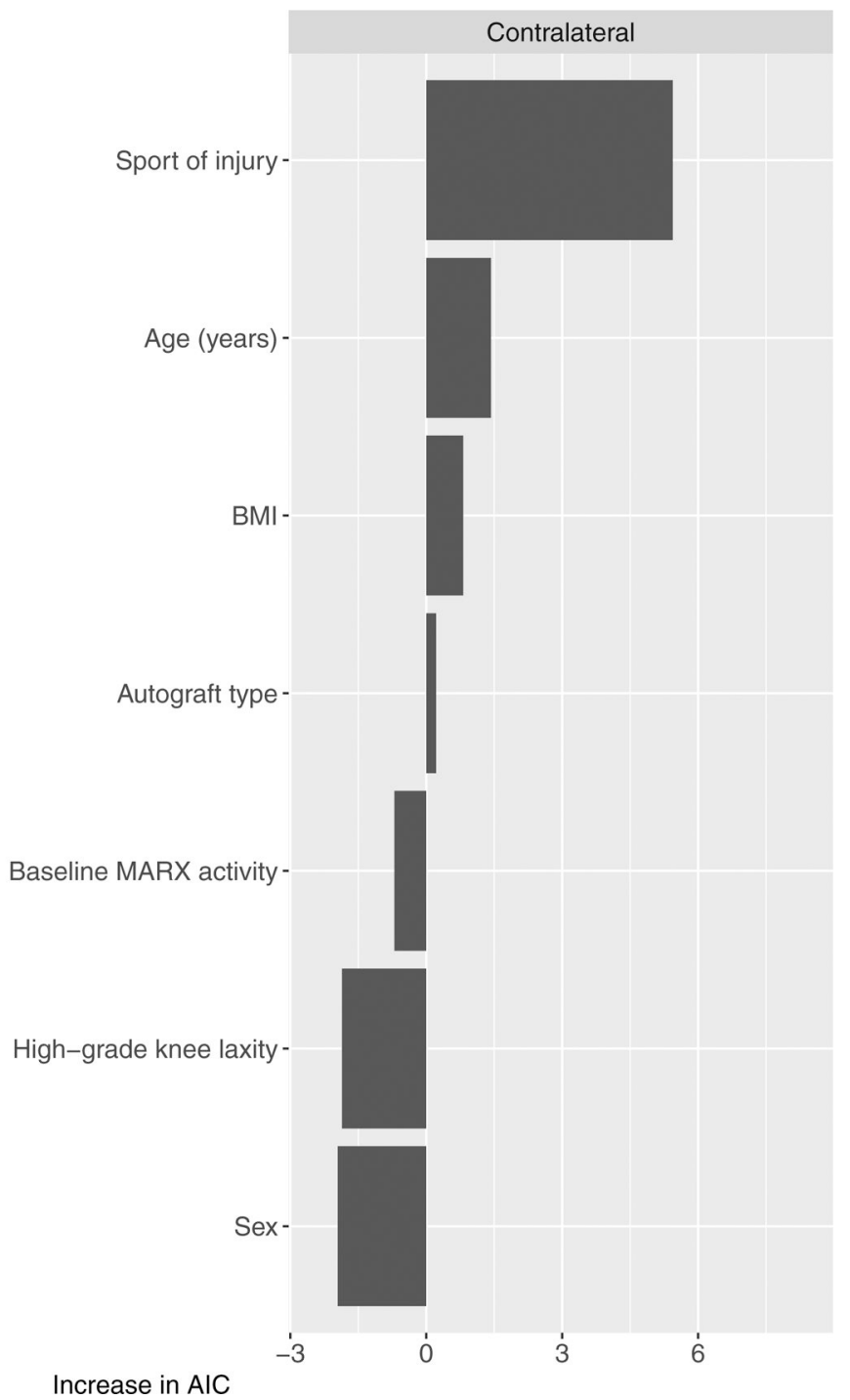

Figure 4.

Relative variable importance for predicting a subsequent ACL reconstruction in the ipsilateral and contralateral knees. 
et al.

Points

Age (years)

BMI

Sex

Sport of

injury

High-grade
knee laxity

Autograft

Baseline

MARX =

16 ?

Total Points
10

20

30

40

50

60

70

Page 19

(a)

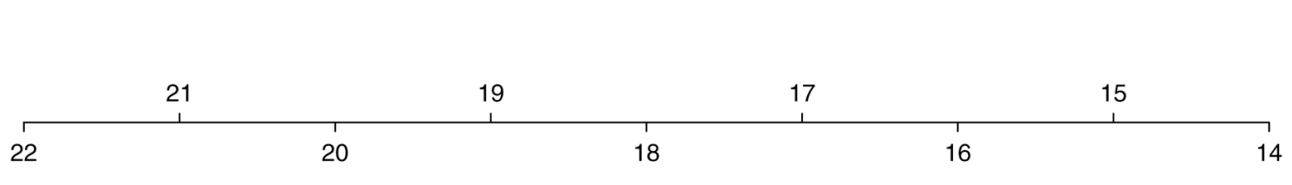

$\begin{array}{lllll}44 & 38 & 32 & 26 & 20\end{array}$
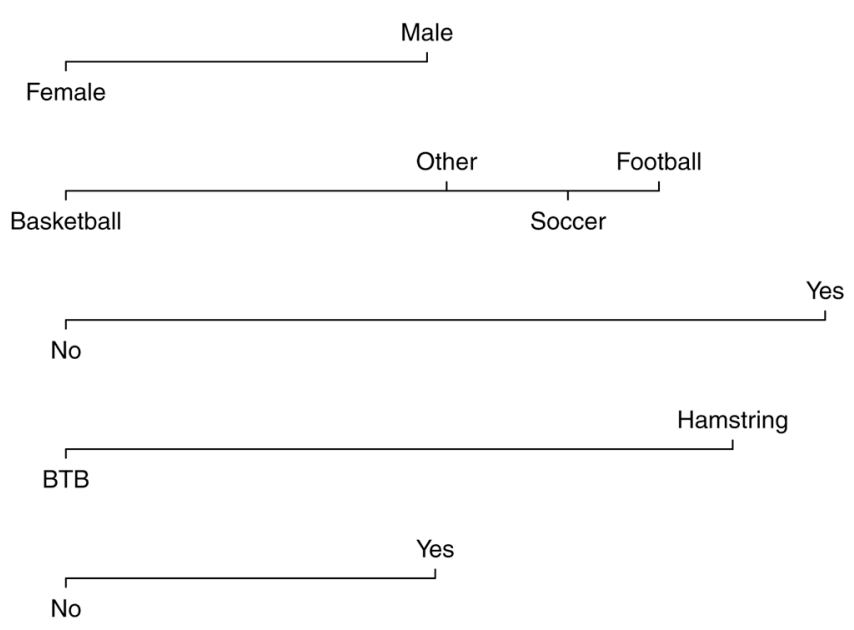

Hamstring

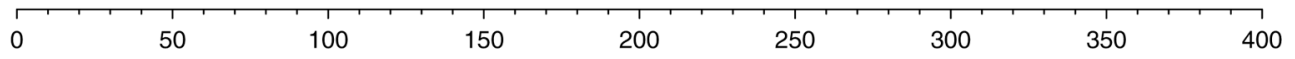

6-year risk of $\mathrm{ACL}$ rupture on ipsilateral knee

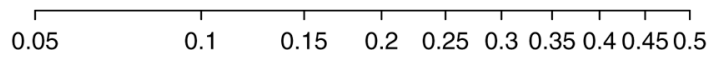

Figure 5.

Nomogram of the logistic regression model used to predict the risk of an ACL graft revision in the ipsilateral knee within 6 years of the index surgery. A link to this online risk calculator can be found on https://www.moonknee.org/calc. This online calculator works on any nonExplorer browser (i.e Chrome, Firefox).

For each athlete, determine the points assigned on the top "points" line for Age, BMI, Sex, Sport of Injury, High-grade knee laxity, Autograft graft type, and baseline Marx activity level. Calculate the patient's total points from all these risk factors and place mark on the "total points" scale on the bottom (0-400). To calculate the 6-year risk, draw a vertical line from the "total points" number down to the predicted percentage risk of failure on the very bottom scale, which ranges from 5 to $50 \%$ (0.05 to 0.5$)$.

Am J Sports Med. Author manuscript; available in PMC 2021 February 01. 
Points

$\begin{array}{llllllllll}0 & 10 & 20 & 30 & 40 & 50 & 60 & 70 & 80 & 90\end{array}$

Age (years)

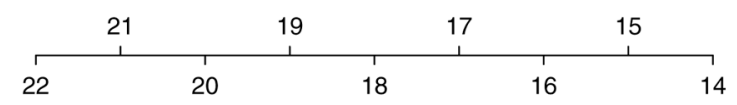

BMI

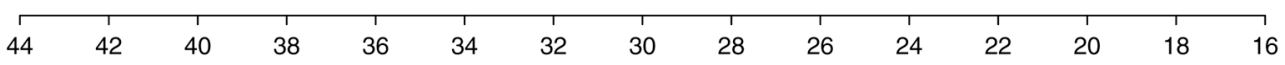

Sex

Female

Male

Sport of

injury

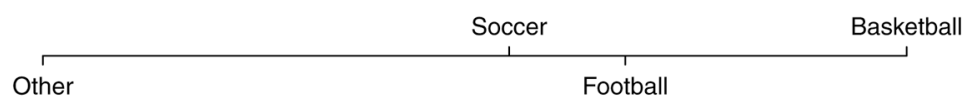

High-grade

knee laxity

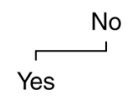

Autograft

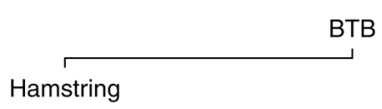

Baseline

MARX =

$16 ?$

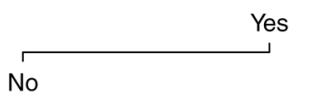

Total Points

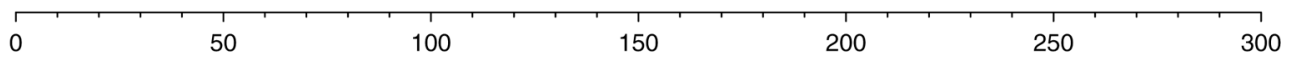

6-year risk of $A C L$ rupture on contralateral knee

\begin{tabular}{lllllll}
\hline 0.05 & 0.1 & 0.15 & 0.2 & 0.25 & 0.3 & 0.35
\end{tabular}

Figure 6.

Nomogram of logistic regression model to predict the risk of a normal ACL tear resulting in a reconstruction in the contralateral knee within 6 years of the index surgery.

For each athlete determine the points assigned on the top "points" line for Age, BMI, Sex, Sport of Injury, High-grade knee laxity, Autograft graft type, and baseline Marx activity level. Calculate the patient's total points from all these risk factors and place mark on the "total points" scale on the bottom (0-300). To calculate the 6-year risk, draw a vertical line from the "total points" number down to the predicted percentage risk of failure on the very bottom scale, which ranges from 5 to $35 \%$ (0.05 to 0.35$)$. 
Table 1.

\section{Baseline Patient Characteristics}

\begin{tabular}{|c|c|c|c|}
\hline & $\begin{array}{c}\text { Entire Cohort } \\
(n=770)\end{array}$ & BTB Autograft only & HS Autograft only \\
\hline Age, years & $17(16,19)$ & $17(16,19)$ & $17(15,19)$ \\
\hline \multicolumn{4}{|l|}{ Sex } \\
\hline - Male & $401(52 \%)$ & $261(53 \%)$ & $140(50 \%)$ \\
\hline - Female & $369(48 \%)$ & $231(47 \%)$ & $138(50 \%)$ \\
\hline \multicolumn{4}{|l|}{ Race/Ethnicity } \\
\hline - White & $642(83 \%)$ & $414(84 \%)$ & $228(82 \%)$ \\
\hline - Black & $76(10 \%)$ & $53(11 \%)$ & $23(8 \%)$ \\
\hline - Other & $52(7 \%)$ & $25(5 \%)$ & $27(10 \%)$ \\
\hline BMI & $23.3(21.3,25.8)$ & $23.6(21.6,26.3)$ & $22.9(21.0,25.6)$ \\
\hline Marx Activity Level (0-16) & $16(15,16)$ & $16(15,16)$ & $16(13,16)$ \\
\hline \multicolumn{4}{|l|}{ Sport at Injury } \\
\hline - Basketball & $222(29 \%)$ & $154(31 \%)$ & $68(25 \%)$ \\
\hline - Football & $180(23 \%)$ & $118(24 \%)$ & $62(22 \%)$ \\
\hline - Soccer & $156(20 \%)$ & $102(21 \%)$ & $54(19 \%)$ \\
\hline - Other & $212(28 \%)$ & $118(24 \%)$ & $94(34 \%)$ \\
\hline \multicolumn{4}{|l|}{ Competition Level } \\
\hline - Recreational & $123(16 \%)$ & $61(12 \%)$ & $62(22 \%)$ \\
\hline - Organized high school/club/college & $646(84 \%)$ & $431(88 \%)$ & $215(77 \%)$ \\
\hline - Not reported & $1(<1 \%)$ & 0 & $1(<1 \%)$ \\
\hline \multicolumn{4}{|l|}{ High-Grade Knee Laxity } \\
\hline$\cdot$ No & $511(66 \%)$ & $318(65 \%)$ & $193(69 \%)$ \\
\hline - males & 280 & 180 & 100 \\
\hline - females & 231 & 138 & 93 \\
\hline - Yes & $257(33 \%)$ & $174(35 \%)$ & $83(30 \%)$ \\
\hline - males & 120 & 81 & 39 \\
\hline - females & 137 & 93 & 44 \\
\hline - Not reported & $2(<1 \%)$ & 0 & $2(<1 \%)$ \\
\hline \multicolumn{4}{|l|}{ Autograft Type } \\
\hline • ВТВ & $492(64 \%)$ & & \\
\hline - Hamstring & $278(36 \%)$ & & \\
\hline
\end{tabular}

Key: continuous variables are reported as medians $(25 \%, 75 \% \mathrm{IQR})$, while categorical variables are reported as counts (percentages). $\mathrm{BTB}=$ bonepatellar tendon-bone; $\mathrm{HS}=$ hamstring. 
Table 2.

Confidence intervals for odds of choosing a BTB versus hamstring autograft

\begin{tabular}{|c|c|c|}
\hline & $95 \% \mathrm{CI}$ & If significant, more likely to receive which autograft? \\
\hline Surgeon & $(2.80,6.54)$ & Dependent upon surgeon \\
\hline \multicolumn{3}{|l|}{ Competition Level } \\
\hline \multicolumn{3}{|l|}{ - Recreational } \\
\hline - Organized high school/club/college & $(0.08,0.48)$ & BTB \\
\hline Age, years & $(0.67,0.88)$ & $\begin{array}{l}\text { Younger age } \\
\text { (BTB) }\end{array}$ \\
\hline \multicolumn{3}{|l|}{ High-Grade Knee Laxity } \\
\hline \multicolumn{3}{|l|}{$\bullet$ No } \\
\hline • Yes & $(0.16,0.71)$ & BTB \\
\hline Body Mass Index (BMI) & $(0.85,0.99)$ & $\begin{array}{c}\text { Lower BMI } \\
\text { (BTB) }\end{array}$ \\
\hline \multicolumn{3}{|l|}{ Race/Ethnicity } \\
\hline \multicolumn{3}{|l|}{ •White } \\
\hline • Black & $(0.10,0.93)$ & BTB \\
\hline - Other & $(0.72,7.09)$ & \\
\hline \multicolumn{3}{|l|}{ Baseline Marx $=16 ?$} \\
\hline \multicolumn{3}{|l|}{$\bullet$ No } \\
\hline • Yes & $(0.32,1.13)$ & \\
\hline \multicolumn{3}{|l|}{ Sex } \\
\hline \multicolumn{3}{|l|}{ - Males } \\
\hline - Females & $(0.34,1.46)$ & \\
\hline \multicolumn{3}{|l|}{ Sport at Injury } \\
\hline \multicolumn{3}{|l|}{ • Basketball } \\
\hline • Football & $(0.28,1.60)$ & \\
\hline - Soccer & $(0.28,1.52)$ & \\
\hline - Other & $(0.59,2.58)$ & \\
\hline
\end{tabular}

Key: bolded values depict variables which are significant. BTB=bone-patellar tendon-bone. 
Table 3.

Incidence of Subsequent ACL Reconstruction at 6 Years

\begin{tabular}{lccr}
\hline & $\begin{array}{c}\text { Entire Dataset } \\
(\mathbf{n = 7 7 0})\end{array}$ & $\begin{array}{c}\text { BTB Autografts } \\
(\mathbf{n = 4 9 2})\end{array}$ & $\begin{array}{c}\text { Hamstring Autografts } \\
(\mathbf{n = 2 7 8})\end{array}$ \\
\hline Subsequent Ipsilateral (IL) ACLR & $71(9.2 \%)$ & $35(7.1 \%)$ & $36(13.0 \%)$ \\
Subsequent Contralateral (CL) ACLR & $86(11.2 \%)$ & $62(12.6 \%)$ & $24(8.6 \%)$ \\
Either IL or CL subsequent ACLR & $152(19.7 \%)$ & $93(18.9 \%)$ & $59(21.2 \%)$ \\
\hline
\end{tabular}

Key: $\mathrm{IL}=$ ipsilateral; $\mathrm{CL}=$ contralateral; $\mathrm{ACLR}=$ anterior cruciate ligament reconstruction; $\mathrm{BTB}=$ bone-patellar tendon-bone 
Table 4.

Odds ratios, 95\% confidence intervals, and p-values for each baseline characteristic for predicting a subsequent ACL reconstruction with 6 years of the index ACL surgery.

\begin{tabular}{|c|c|c|c|c|}
\hline Variable & $\begin{array}{l}\text { Ipsilateral Knee } \\
\text { OR }(95 \% \text { CI })\end{array}$ & p-value & $\begin{array}{c}\text { Contralateral Knee } \\
\text { OR }(95 \% \text { CI })\end{array}$ & p-value \\
\hline Age & $0.84(0.74,0.96)$ & 0.009 & $0.89(0.79,1.01)$ & 0.068 \\
\hline BMI & $0.99(0.92,1.06)$ & 0.791 & $0.94(0.88,1.01)$ & 0.107 \\
\hline \multicolumn{5}{|l|}{ Sex } \\
\hline \multicolumn{5}{|l|}{ - Male } \\
\hline - Female & $0.67(0.35,1.26)$ & 0.211 & $1.06(0.6,1.88)$ & 0.833 \\
\hline \multicolumn{5}{|l|}{ Sport of Injury } \\
\hline \multicolumn{5}{|l|}{ - Basketball } \\
\hline - Football & $1.95(0.87,4.38)$ & 0.107 & $0.69(0.34,1.41)$ & 0.307 \\
\hline - Soccer & $1.76(0.82,3.77)$ & 0.147 & $0.59(0.32,1.09)$ & 0.092 \\
\hline - Other & $1.53(0.72,3.29)$ & 0.272 & $0.32(0.16,0.65)$ & 0.002 \\
\hline \multicolumn{5}{|c|}{ High-Grade Knee Laxity } \\
\hline \multicolumn{5}{|l|}{$\cdot$ No } \\
\hline • Yes & $2.35(1.4,3.93)$ & 0.001 & $0.91(0.56,1.48)$ & 0.708 \\
\hline \multicolumn{5}{|l|}{ Autograft Type } \\
\hline \multicolumn{5}{|l|}{$\bullet$ ВТВ } \\
\hline - Hamstring & $2.12(1.27,3.54)$ & 0.004 & $0.68(0.41,1.14)$ & 0.144 \\
\hline \multicolumn{5}{|c|}{ Baseline Marx $=16 ?$} \\
\hline \multicolumn{5}{|l|}{ • No } \\
\hline • Yes & $1.51(0.82,2.79)$ & 0.182 & $1.39(0.78,2.47)$ & 0.265 \\
\hline
\end{tabular}

Am J Sports Med. Author manuscript; available in PMC 2021 February 01. 